\title{
HIERARCHICAL AGGLOMERATIVE CLUSTERING SCHEMES FOR ENERGY-EFFICIENCY IN WIRELESS SENSOR NETWORKS
}

\author{
Tariq Taleb ${ }^{1}$, Mejdi Kaddour ${ }^{2}$ \\ Laboratoire d'Informatique et des Technologies d'Information d'Oran - LITIO \\ Computer Science Department, University of Oran 1 Ahmed BenBella \\ B.P. 1524, EL-M'nouar Oran - Algeria \\ E-mails: ${ }^{1}$ tariq.taleb8@gmail.com, ${ }^{2}$ kaddour.mejdi@univ-oran.dz
}

\begin{abstract}
Extending the lifetime of wireless sensor networks (WSNs) while delivering the expected level of service remains a hot research topic. Clustering has been identified in the literature as one of the primary means to save communication energy. In this paper, we argue that hierarchical agglomerative clustering (HAC) provides a suitable foundation for designing highly energy efficient communication protocols for WSNs. To this end, we study a new mechanism for selecting cluster heads (CHs) based both on the physical location of the sensors and their residual energy. Furthermore, we study different patterns of communications between the $\mathrm{CHs}$ and the base station depending on the possible transmission ranges and the ability of the sensors to act as traffic relays. Simulation results show that our proposed clustering and communication schemes outperform well-knows existing approaches by comfortable margins. In particular, networks lifetime is increased by more than $60 \%$ compared to LEACH and HEED, and by more than $30 \%$ compared to K-means clustering.
\end{abstract}

Keywords: WSNs, hierarchical agglomerative clustering, cluster head, energy-efficiency

\section{Introduction}

Wireless sensor networks (WSNs) are designed to be used in a wide set of applications related to various domains, such as science, logistics, military or health. According to Dong and Chun (2012) in the MIT's Technology Review, WSNs are one of the ten technologies that will change the world and the way we live and work.

The battery is a critical element of a wireless sensor. In general, it is not replaceable or rechargeable, but it may be partly supplied by a power generating unit such as solar cells.

Due to its size limitation, it provides a very limited amount of energy on the range of 1 to 2 Joule per sensor node cited in Heinzelman et al. (2002). Hence, the overall network lifetime can be severely affected. Therefore, the protocols which contribute to save energy have received much attention from the community.

Among the several tasks performed by a sensor, such as data acquisition or processing, communication is widely considered as the most energy-consuming. An efficient energy management scheme should, as a priority, considers the consumption of energy induced by communication. However, most communication protocols designed for wireless multi-hop networks do not adapt to the characteristics of WSNs. Hence, there is an absolute need to improve or develop new protocols. Most protocols used today in wireless sensor networks are typically based on some combination of multi-hop routing and clustering.

In the context of routing, several approaches have been proposed to calculate the optimal path towards the sink or the base station (BS) for each sensing node. A first category of such protocols proposes to select the shortest path in term of distance to the base station according to The World. Technology review (2003). Whereas, other protocols are based on the energy level at the nodes along the path by selecting first the nodes having highest amounts of energy. A third category favours the paths constituted by the nodes which will consume less energy. However, all these flat routing protocols share an important shortcoming which lies in the frequency of control messages sent to maintain valid routes. This may have severe effects on network congestion and energy consumption according to Youn and Kim (2005).

In this paper, we design a clustering scheme based on hierarchical agglomerative clustering in Lukasová (1979). Unlike some existent approaches based on probabilistic decisions, such as LEACH by Heinzelman et al. (2002), our scheme is deterministic. Also, we introduce a new cluster head (CH) selection algorithm, based on the proximity with a virtual node representing the optimal $\mathrm{CH}$ location with respect both to the geometric centroid of the cluster and energy consumption. 
Furthermore, depending on the transmission characteristics of sensor nodes, we propose three different schemes to convey data traffic from the $\mathrm{CHs}$, which gather all the sensing data transmitted by their associated nodes, to the BS. These schemes are:

- SH-HAC: direct single-hop communication between the $\mathrm{CH}$ and the BS;

- MH-HAC: multi-hop communication between the $\mathrm{CHs}$ and the $\mathrm{BS}$, where the $\mathrm{CHs}$ are assumed to have unlimited transmission range.

- $\quad$ RL-HAC: similar to MH-HAC but CHs have limited transmission range.

The rest of this paper is organized as follows. Section 2 describes related works and in particular the existing representative cluster-based routing protocols proposed for WSNs.

For the sake of self-containment, Section 3 gives a brief overview of some concepts that we rely on: energy consumption model and hierarchical agglomerative clustering.

Section 4 describes our proposed approaches based on hierarchical agglomerative clustering. We evaluate in Section 5 several simulation results, and make comparisons with three alternative clustering solutions: LEACH by Heinzelman et al. (2002), HEED by Younis and Fahmy (2004) and K-means clustering Park et al. (2013). Finally, conclusions are drawn in Section 6.

\section{Related Work}

There are many works in the literature proposing various approaches and protocols to support energy-aware communication in WSNs. In this section, we focus only on the hierarchical type of protocols.

In 2000, Heizelman introduced the very-known hierarchical routing algorithm named LEACH in (Heinzelman et al., 2002). Its key idea is that every sensor node can be elected as $\mathrm{CH}$ according to the probability calculated by whether it has been already elected as $\mathrm{CH}$ or not. In the clustering phase, a number between 0 and 1 is randomly selected by each sensor node. If this number is less than a unified calculated threshold, the node would become the $\mathrm{CH}$; otherwise, it selects a cluster to join in. This threshold is obtained by (1) where $P$ represents the desired proportion of CHs in the WSN.

$T(n)=\left\{\begin{array}{cl}\frac{P}{1-P\left(r \bmod \frac{1}{P}\right)} & n \in G \\ 0 & \text { otherwise }\end{array}\right.$

Afterwards, each $\mathrm{CH}$ broadcasts an inviting message to other nodes. Then, each receiving node determines which $\mathrm{CH}$ to join according to the corresponding received signal strengths. Note that LEACH triggers re-clustering and $\mathrm{CH}$ rotation in a periodic fashion to improve the energy-efficiency of the network. Some experiments have shown that LEACH achieves significant gains in network lifetime, but its main drawback is that residual energy is not considered in the $\mathrm{CH}$ selection process.

LEACH-C by Heinzelman et al. (2002), adopts a more centralized approach as the sink is responsible for choosing the $\mathrm{CHs}$ by collecting all sensor nodes information. The advantage of this method is that a global view of the energy budget is available when taking decisions, but it suffers from serious limitations as all the nodes are required to communicate directly with the sink.

Furthermore, farthest nodes have to consume more energy than the nearest ones because more transmission power is necessary. Thus, the network scalability may be affected significantly.

Alternatively, HEED chooses the CHs by looking to the residual energy of each member node. It also considers the inter-cluster communication cost as a secondary parameter. Its algorithm consists in the three following phases. In the first phase, each node identifies its neighbours and then computes the energy required for communicating with them. It also calculates the primary $\mathrm{CH}$ selection probability using:

$C H_{\text {prob }}=C_{\text {prob }} \times \frac{E_{\text {residual }}}{E_{\text {max }}}$,

where $C_{\text {prob }}$ is the initial percentage of $\mathrm{CHs}$ among the total nodes, $E_{\text {residual }}$ is the estimated residual energy of the node, and $E_{\max }$ is the reference maximum energy. In the second phase each node broadcasts an advertisement message (ADV) to other nodes of its cluster and receives the ADV messages from other candidate $\mathrm{CHs}$. The ADV message contains the values obtained in the first phase. Candidate CHs cancel their candidacy if their communication cost is larger than other candidates. In the last phase, 
the non- $\mathrm{CH}$ nodes select a $\mathrm{CH}$ to join in. Here, if some nodes have the same $C_{\text {prob }}$ value, the one with the smallest average minimum reachability power obtained with (3) is selected as a $\mathrm{CH}$.

$A M R P=\frac{\sum_{i=0}^{M} P r_{i}}{M}$,

where $M$ is the number of nodes in the cluster and $\operatorname{Pr}_{i}$ is the minimum power required to transmit to node $i$. In summary, HEED protocol results in a slightly different cluster structure from LEACH-kind protocols.

TEEN of Manjeshwar and Agrawal (2001), is similar to LEACH, but proposes a hard threshold and a soft threshold to reduce the number of control messages. However, the values of these thresholds are difficult to set. Hard threshold tries to reduce the number of transmissions by allowing the nodes to transmit only when the sensed attribute is in the range of interest. Soft threshold further reduces the number of transmissions by eliminating all the transmissions which might have otherwise occurred when there is little or no change in the sensed attribute. ERA of Chen et al. (2007), is another hierarchical algorithm inspired by LEACH. Its originality lies in the inclusion of the communication costs in the clustering process. The communication cost of a $\mathrm{CH}$ consists of nodes' residual energy, the distance from the sink node and the distance from other cluster's members. Still that this algorithm does not consider current residual energy in the re-clustering procedure.

All the algorithms presented above adopt a dynamic clustering approach, in which $\mathrm{CHs}$ are chosen before the clusters have been actually constituted. After a few transmission rounds, a re-clustering procedure is necessary to balance energy consumption in the WSN, but this in turn consumes a nonnegligible amount as many control messages are needed. For this reason, a set of hierarchical routing algorithms took up a more static clustering approach, where nodes are first clustered and then $\mathrm{CHs}$ elected. Hence, the cluster composition remains unchanged for the whole network lifetime. Only the $\mathrm{CH}$ responsibility can be turned over after some communication rounds. Park in (2013) designed a $\mathrm{CH}$ selection method based on the K-means clustering (CHSMK-means). Here, the nearest node to the cluster centroid with sufficient energy is elected as a $\mathrm{CH}$. Results showed that this selection mechanism leads to better performance in terms of network lifetime and energy efficiency compared to LEACH. In Lung and Zhou (2010), a clustering algorithm named DHAC (Distributed HAC) was introduced.

In DHAC, not only the sensor nodes are clustered one and for all in the initial stage, but also an order of $\mathrm{CH}$ rotation is figured out at this stage. This has an obvious advantage in energy conservation than dynamic clustering schemes. However, residual node energy is also not taken into account and cluster structure can be too rigid to cope with changing conditions, such as node failures or radio transmission disruptions used by Du et al. (2015).

\section{Background}

\section{a. Energy Consumption Model}

We review in this subsection some classical formulas to estimate the energy dissipation in radio communication used by Heinzelman et al. (2002). The energy required for transmitting a data packet of $k$ bits at some distance $d$ is given as:

$E_{T x}(k, d)=\left\{\begin{array}{ll}E_{\text {elec }} \times k+\varepsilon_{f s} \times k \times d^{2}, & d<d_{0} \\ E_{\text {elec }} \times k+\varepsilon_{m p} \times k \times d^{4}, & d \geq d_{0}\end{array}\right.$,

where $E_{\text {elec }}$ is a physical parameter depending on the transmission channel, $\varepsilon_{f s}$ and $\varepsilon_{m p}$ are also physical parameters representing energy consumption in the amplifier circuitry, and $d_{0}$ is a reference distance related to node's hardware. This latter can be given as:

$d_{0}=\sqrt{\frac{\varepsilon_{f s}}{\varepsilon_{m p}}}$.

From (4), it is easy to see that distance and data packet size are the key factors that affect energy consumption as most sensors have comparable physical parameters. In this paper, we focus on working around the first factor. 


\section{b. Hierarchical agglomerative clustering}

Hierarchical agglomerative clustering (HAC) consists in grouping data points (or network nodes) iteratively one by one on the basis of the smallest distance measure over all the pairwise distances between the data points. The distances between the formed clusters are recalculated at each iteration. Several distance measures were proposed in the literature, such as:

- $\quad$ Single-nearest distance or single linkage.

- Complete-furthest distance or complete linkage.

- Average-average distance or average linkage.

- Centroid distance.

- Ward's method: sum of squared Euclidean distance is minimized.

Data grouping proceeds until a single big cluster is formed. Now on the basis of a Dendrogram graph we can calculate how many number of clusters should be actually present.

Formally, we can state the HAC algorithm in the following steps:

1. Begin with the disjoint clustering having level $L(0)=0$ and sequence number $m=0$.

2. Find the least distance pair of clusters in the current clustering, say pair $(r),(s)$, according to $d[(r),(s)]=\min d[(i),(j)]$ where the minimum is over all pairs of clusters in the current clustering.

3. Increment the sequence number; merge clusters $(r)$ and $(s)$ into a single cluster to form the next clustering $m$; Set the level of this clustering to $L(m)=d[(r),(s)]$.

4. Update the distance matrix $D$, by deleting the rows and columns corresponding to clusters $(r)$ and $(s)$ and adding a row and column corresponding to the newly formed cluster. The distance between the new cluster, denoted $(r, s)$ and an existing cluster $(k)$ is defined as : $d[(k),(r, s)]=$ $\min d[(k),(r)], d[(k),(s)]$.

5. If all the data points are in one cluster then stop, else repeat from step 2.

Among the advantages of HAC, we may notice that no a priori information about the number of clusters is required, and its ease of implementation.

An illustrative example

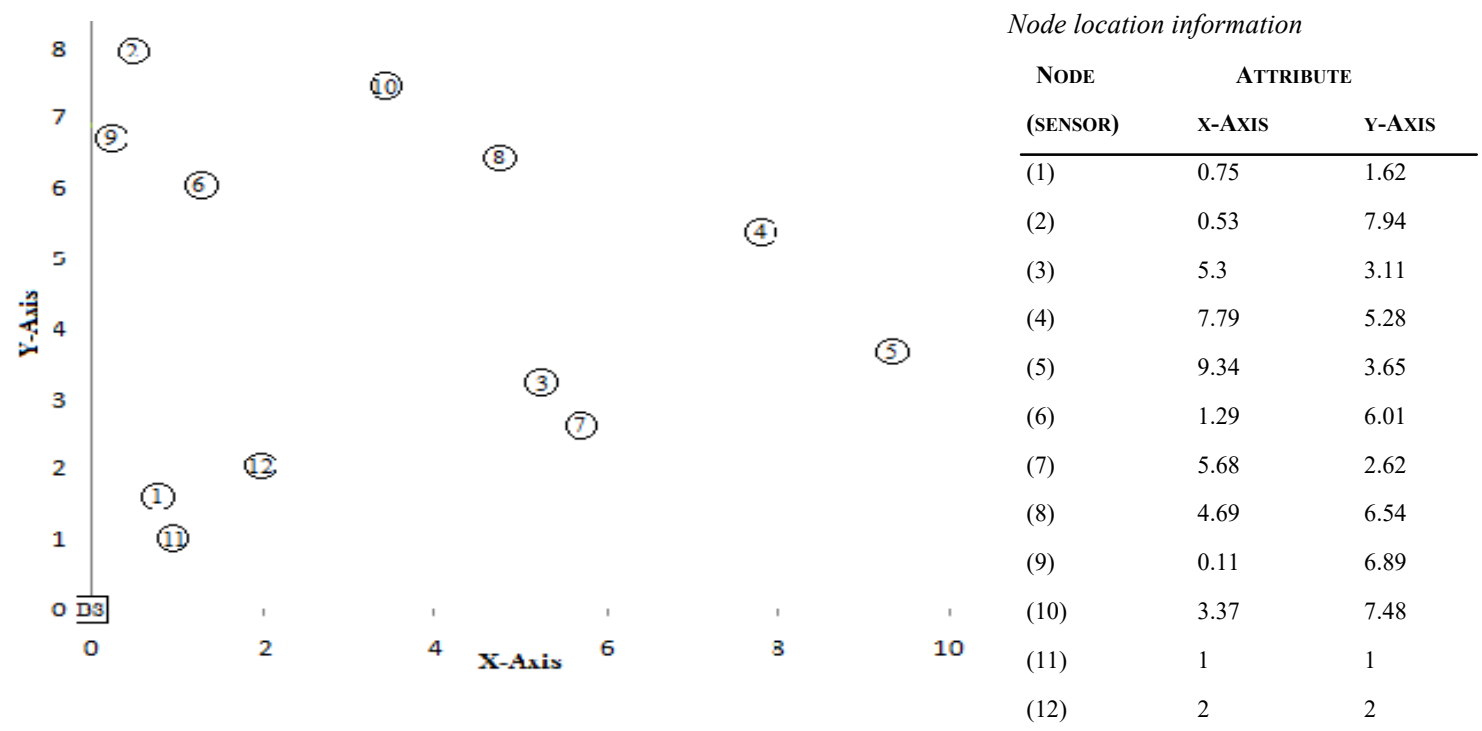

Figure. 1. A 12-node network topology

Figure 1 shows a randomly generated 12 -node network within a $10 \times 10 \mathrm{~m}^{2}$ field, along with the location information of each node. The distance between each node in the network is calculated using the Euclidean distance as represented in Table 1.

The distance measure for cluster merging is the single-nearest distance. The dissimilarity matrix is updated accordingly until obtaining one cluster. After the completion of the HAC, we obtain the dendrogram of Figure 2. As it can be seen, the best score is 2.54 in the index hierarchy and includes 4 clusters: $\mathrm{C} 1=\{1,11,12\}, \mathrm{C} 2=\{2,6,8,9,10\}, \mathrm{C} 3=\{3,7\}$ and $\mathrm{C} 4=\{4,5\}$. If two among these clusters are further merged, we would get a sudden change in the index hierarchy (smallest distance between clusters) from 2.54 to 3.39 . 
Table 1. The distance of every pair of nodes (Dissimilarity matrix) using Euclidean distance

\begin{tabular}{|c|c|c|c|c|c|c|c|c|c|c|c|c|}
\hline & (1) & (2) & (3) & (4) & (5) & (6) & (7) & (8) & (9) & (10) & (11) & (12) \\
\hline$(1)$ & 0 & & & & & & & & & & & \\
\hline (2) & 6.32 & 0 & & & & & & & & & & \\
\hline (3) & 4.78 & 6.78 & 0 & & & & & & & & & \\
\hline (4) & 7.93 & 7.73 & 3.3 & 0 & & & & & & & & \\
\hline (5) & 8.82 & 9.79 & 4.07 & 2.24 & 0 & & & & & & & \\
\hline (6) & 4.42 & 2.07 & 4.94 & 6.54 & 8.38 & 0 & & & & & & \\
\hline (7) & 5.03 & 7.4 & 0.62 & 3.39 & 3.8 & 5.54 & 0 & & & & & \\
\hline (8) & 6.3 & 4.38 & 3.48 & 3.34 & 5.47 & 3.44 & 4.04 & 0 & & & & \\
\hline (9) & 5.3 & 1.13 & 6.42 & 7.84 & 9.78 & 1.47 & 7.01 & 4.59 & 0 & & & \\
\hline (10) & 6.41 & 2.87 & 4.77 & 4.93 & 7.09 & 2.54 & 5.38 & 1.62 & 3.31 & 0 & & \\
\hline (11) & 0.66 & 6.95 & 4.78 & 8.02 & 8.75 & 5.01 & 4.94 & 6.65 & 5.95 & 6.89 & 0 & \\
\hline (12) & 1.3 & 6.11 & 3.48 & 6.65 & 7.52 & 4.07 & 3.73 & 5.27 & 5.24 & 5.64 & 1.41 & 0 \\
\hline
\end{tabular}

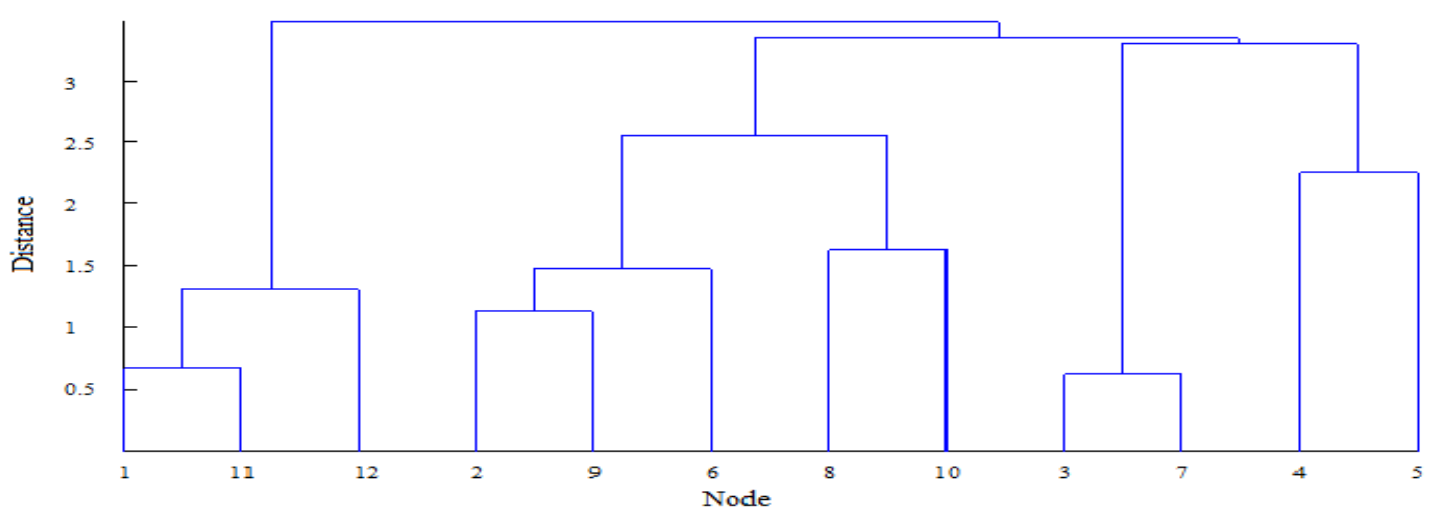

Figure 2. Dendrogram (HAC algorithm)

\section{Proposed HAC- based schemes for WSNs}

We introduce first a set of assumption behind our clustering schemes for WSNs:

- Each node has a unique ID;

- Nodes are fixed and know their geographical positions by some means, such GPS;

- Nodes have the same initial energy;

- $\mathrm{CH}$ nodes are aware of their remaining energy.

We present our schemes in the next three subsections.

\section{a. Single-Hop Hierarchical Agglomerative Clustering (SH-HAC)}

The SH-HAC algorithm is based on the traditional HAC for the formation of the clusters of nodes. The adopted similarity measure is the nearest neighbour in terms of Euclidean distance. Unlike the Kmeans clustering where the number of cluster is given as an input, the number of resulting clusters is based on a sudden change criteria as shown in the example of Section 3.2. The selection of the cluster heads (CHs) obeys to the following three steps:

Initial Clustering, Re-Clustering, CH selection, as described hereafter. Note that this overall procedure is re-executed after a predefined number of communication rounds between the sensor nodes and the BS.

Initial Clustering: Let assume that we obtain $k$ clusters from HAC. For each cluster, the $\mathrm{CH}$ selected initially is the closest one to the BS.

Re-Clustering: Now that every node is associated to one of the $\mathrm{k}$ clusters, we calculate for each cluster a virtual position opt having the following coordinates:

$X_{\text {opti }}=\frac{1}{\sum_{i=1}^{S} E_{i}} \times \sum_{j=1}^{S} E_{j} x_{j}$,
$Y_{\text {opti }}=\frac{1}{\sum_{i=1}^{S} E_{i}} \times \sum_{j=1}^{S} E_{j} y_{j}$, 
where $\left(x_{i}, y_{i}\right)$ and $E_{i}$ refer to the coordinates of the i-th node in the cluster and its residual energy, respectively. Note that the virtual point opt will be located at the centroid of the corresponding cluster as

all the member nodes have the same initial amount of energy.

CH selection: Among the cluster members, the selected $\mathrm{CH}$ is the one having the smallest Euclidean distance to the opt point defined previously. As the network operates, the opt point moves towards the nodes having the highest amounts of residual energy.

Consequently, the new $\mathrm{CH}$ will be selected among theses nodes. This rule is devised to extend the network lifetime as $\mathrm{CHs}$ dissipate more energy by forwarding the traffic to the BS.

Figure 3 illustrates an example where the selected $\mathrm{CHs}$ of the four obtained clusters are depicted in red.

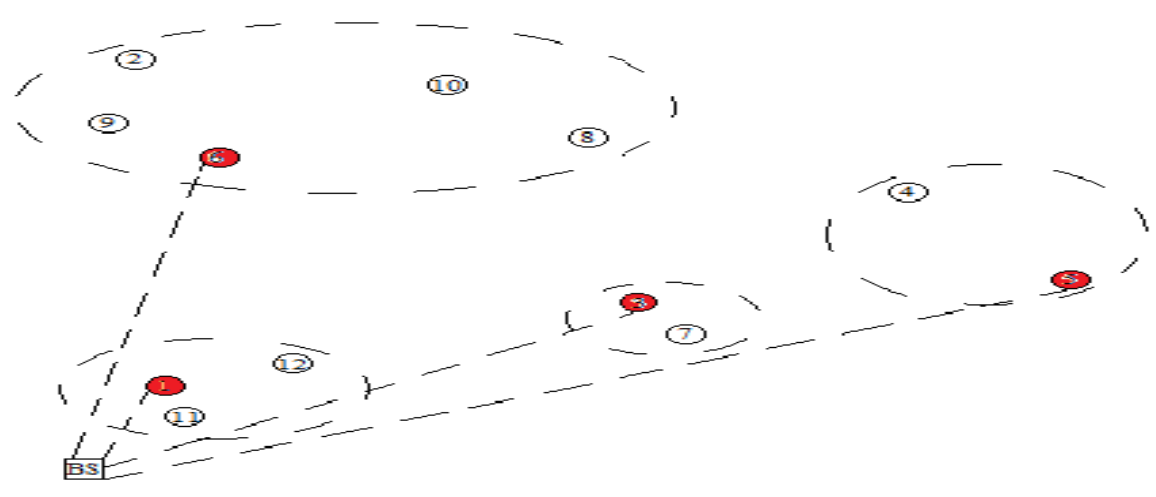

Figure 3. Single hop routing for data transmission

\section{b. Multi-Hop Hierarchical Agglomerative Clustering (MH-HAC)}

$\mathrm{MH}-\mathrm{HAC}$ follows the same steps as SH-HAC, except that the $\mathrm{CHs}$ are no more restricted to send their data directly to the BS. Hence, CHs can also act as relays for data traffic coming from other clusters. The pursued advantage is to minimize CH's transmission cost, which increases dramatically as a function of distance as shown by (4). Our strategy is to build a minimum spanning tree between the BS and the selected $\mathrm{CHs}$, where the weight of each edge corresponds to the Euclidean distance between the two ends. We rely here on the Prim's algorithm in (WeiWang et al., 2014), which we provide here for the sake of completeness.

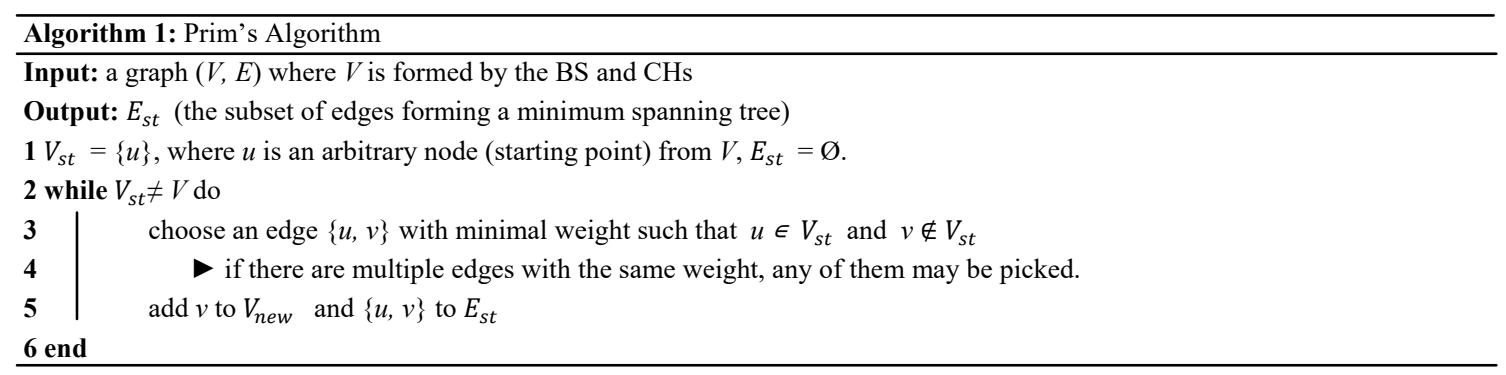

An illustrative Example
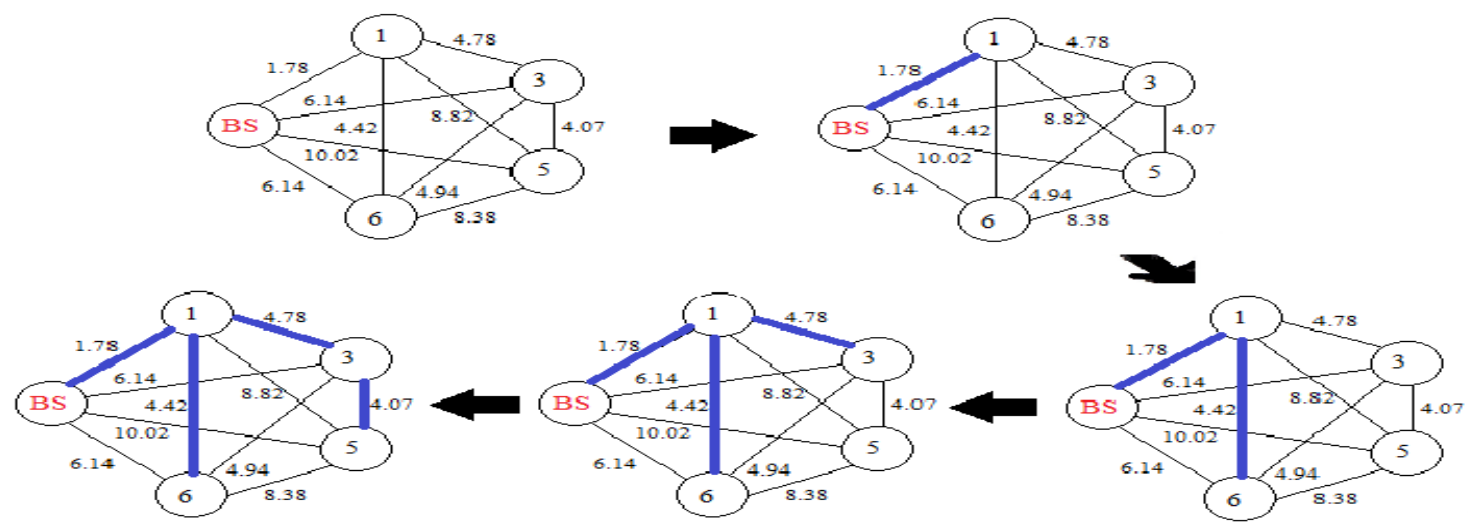

Figure 4. An execution example of Prim's Algorithm 
Figure 4 represents a network with 4 clusters, where we calculated the distance between all CHs and BS. In our algorithm, the root of the tree is always the BS and the edges' weights correspond to the distances between the nodes.

Now, each $\mathrm{CH}$ sends data packets to the BS or other $\mathrm{CH}$ s according to the obtained spanning tree.

\section{c. Range-Limited Hierarchical Agglomerative Clustering (RL-HAC)}

The previous schemes assume implicitly that transmission range is unlimited. That means that every node in the networks can reach directly any other node by setting enough transmission power. This is not the case in real-world applications, where real sensors, such as those equipped with IEEE 802.15.4 transceivers, are range-limited. Thus, SH-HAC and MH-HAC are not applicable with this kind of technology. To cope with this restriction, we introduce in this subsection a third clustering scheme called Range-Limited HAC (RLHAC).

RL-HAC executes the HAC phase by enforcing two more conditions:

- A sensor can transmit a data packet only to another nodes located at a distance not greater than $d$.

- To merge two clusters, there must be at least one member node which is able to communicate directly with the other members of the two clusters.

Algorithm 2 describes formally the merging procedure.

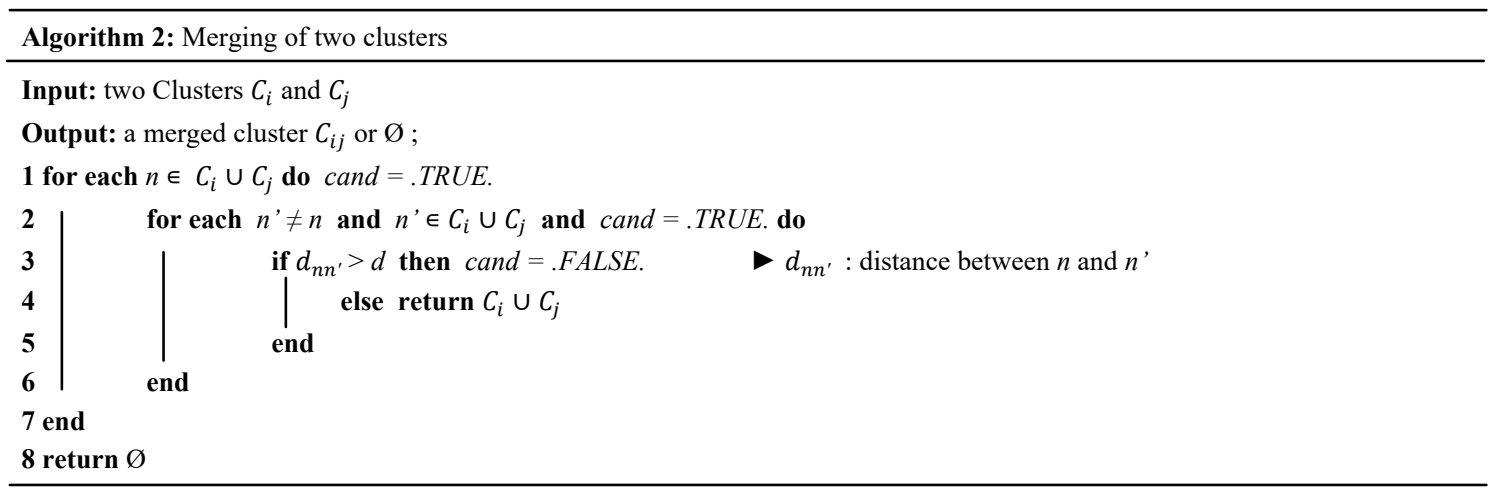

Now, the selected CHs after the clustering phase may not form a spanning tree with the BS as in the previous case. Due to the transmission range limitation, there is no guarantee that a $\mathrm{CH}$ will be able to transmit data to the $\mathrm{BS}$ or to another $\mathrm{CH}$. Consequently, to ensure connectivity among the $\mathrm{CHs}$ and the $\mathrm{BS}$, we may rely on some regular cluster members to act as data relays when direct communication is not possible. This corresponds in fact to the Euclidean Steiner tree problem which was proved to be NP-hard approved by Robins and Zelikovsky (2005). However, there exists a polynomial-time algorithm of Kou et al. (1981), which solves this problem with a constant approximation factor of 2. Algorithm 3 transposes this algorithm to our problem.

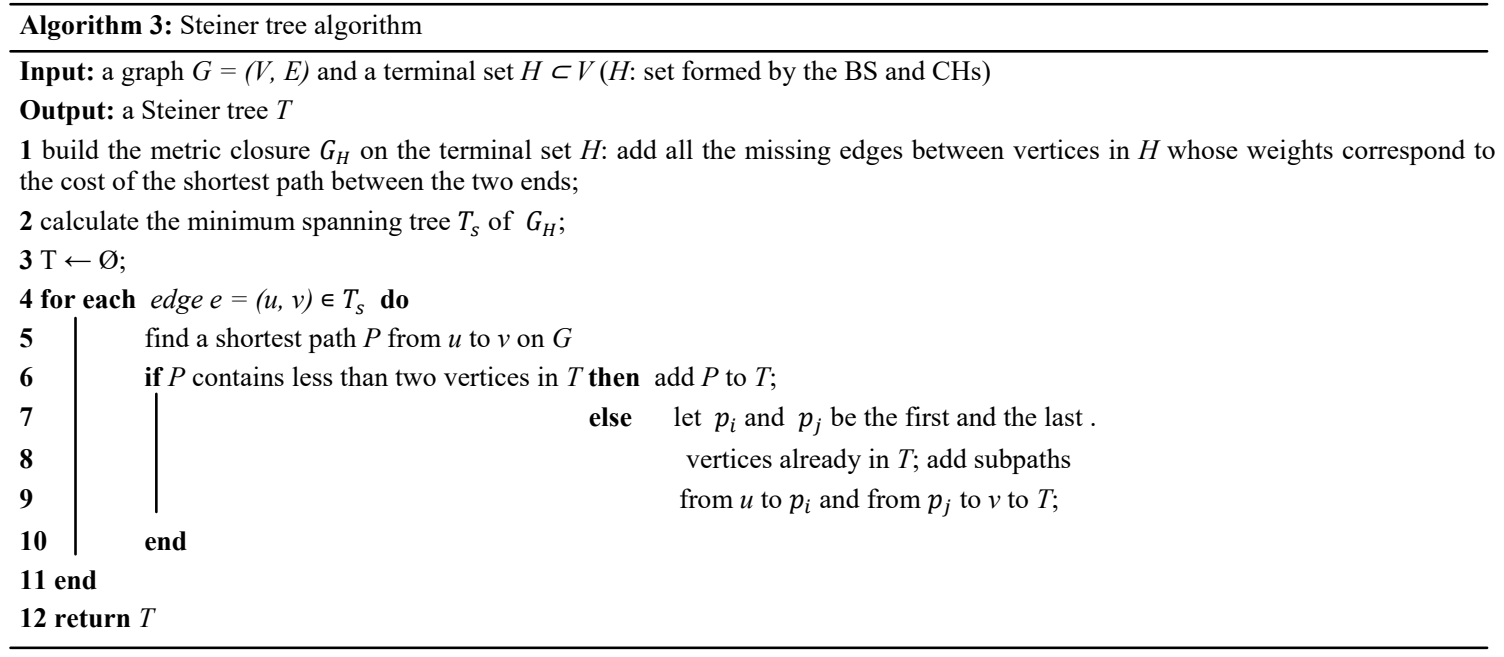




\section{An Illustrative Example}

Figure 5 represents the same WSN instance of Figure 3, where the maximum transmission range is set to some given value. The dashed lines represent the possible transmission links. Based on the Dendrogram of Figure 2 and the similarity matrix in Table 1, we obtain the 3 following clusters: $C 1=\{1$, $11,12\}, C 2=\{2,6,8,9,10\}$ and $C 3=\{3,4,5,7\}$. At this point, the RL-HAC cannot continue to merge any further clusters. For example, node 2 of cluster 2 cannot establish a communication link with node 5 of cluster 3 because the separating distance is beyond the limit.

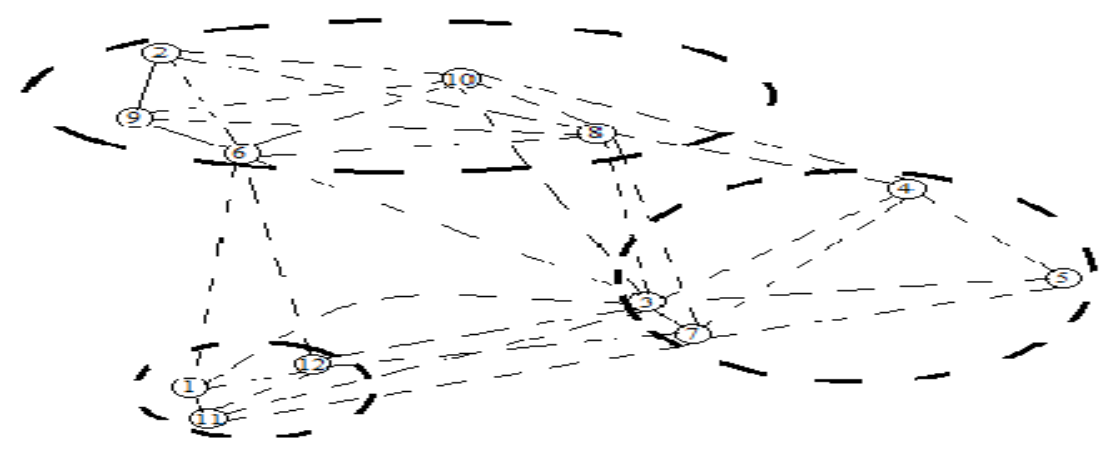

Figure 5. An example of the RL-HAC scheme

Figure 6 shows the successive steps to obtain the Steiner tree. The CHs are nodes 1, 6 and 7. As we can see, node 11 is added to the tree to act as a relay between node 7 and the BS.

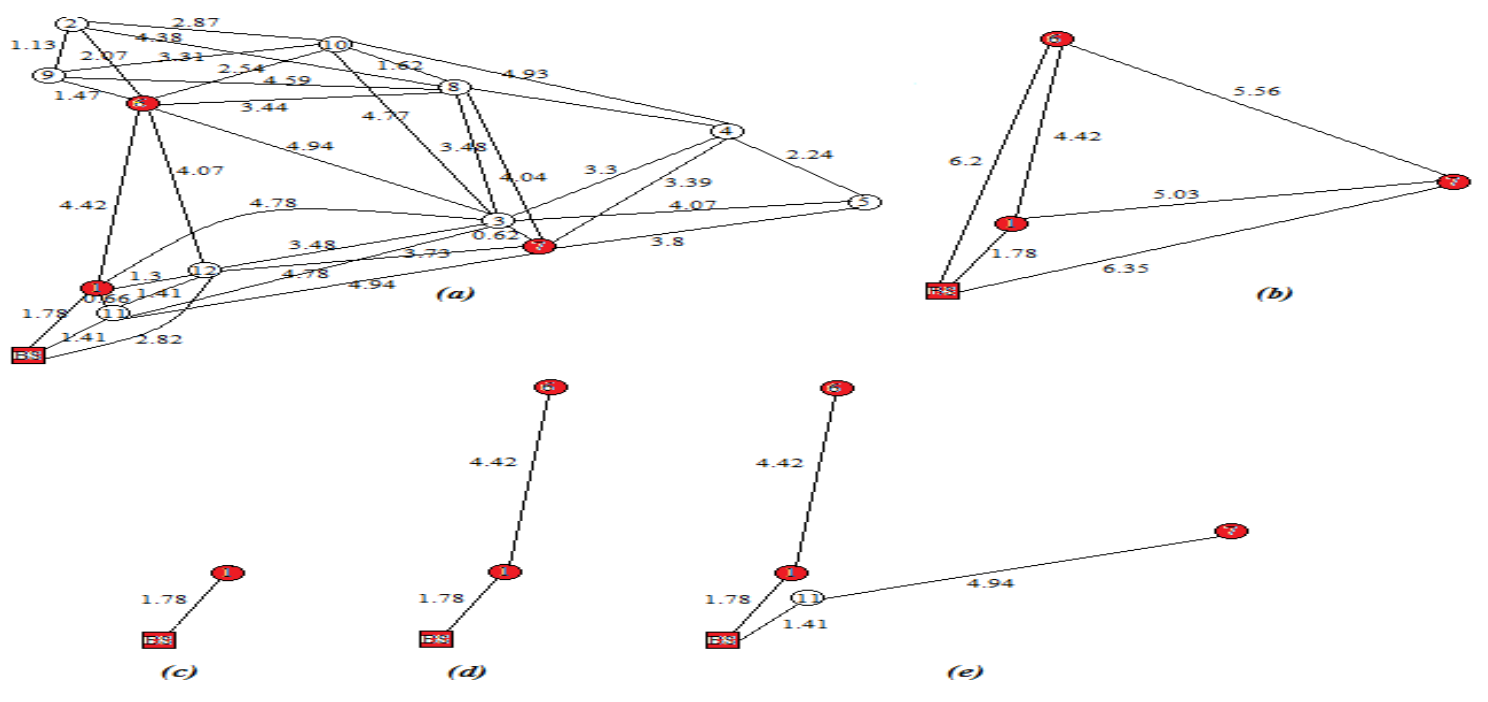

Figure 6. An execution example of the Steiner tree algorithm

\section{Simulation Results}

The conducted experiments in order to validate and to evaluate the quality of the solutions obtained by our clustering schemes were described in this section. A WSN composed from 200 sensors, randomly deployed over a area of $200 \mathrm{~m}$ x $200 \mathrm{~m}$ was considered. Other simulation parameters are summarized in Table 2. We compare our three clustering schemes SHHAC, MH-HA, RL-HAC with LEACH, HEED and CHSMK-means. In order to experiment these various schemes, we have implemented a special purpose simulator using Java language. In particular, this simulator implements the energy consumption model described in Section 3.1. We measure the number of transmission rounds until the energy depletion of all the sensors. Note that each round corresponds to the transmission of one data packet from each sensor to the BS. Besides, we provide as an input to LEACH, HEED and CHSMKmeans the number of clusters that return the best results in terms of network lifetime. In our case, the number of clusters is self-devised through HAC. 
Table 2. Simulation parameters

\begin{tabular}{cc}
\hline Parameter & Value \\
\hline BS location & $(0,0)$ \\
Initial sensor energy & $2 \mathrm{~J}$ \\
$E_{\text {elec }}$ & $10 \mathrm{~nJ} / \mathrm{bit}$ \\
$\varepsilon_{f s}$ & $10 \mathrm{pJ} / \mathrm{bit} / \mathrm{m}^{2}$ \\
$\varepsilon_{m p}$ & $0.0013 \mathrm{pJ} / \mathrm{bit}^{\mathrm{m}} \mathrm{m}^{2}$ \\
$d_{0}$ & $88 \mathrm{~m}$ \\
Data packet size & $500 \mathrm{byte}$ \\
Maximum transmission distance for RL-HAC & $100 \mathrm{~m}$ \\
\hline
\end{tabular}

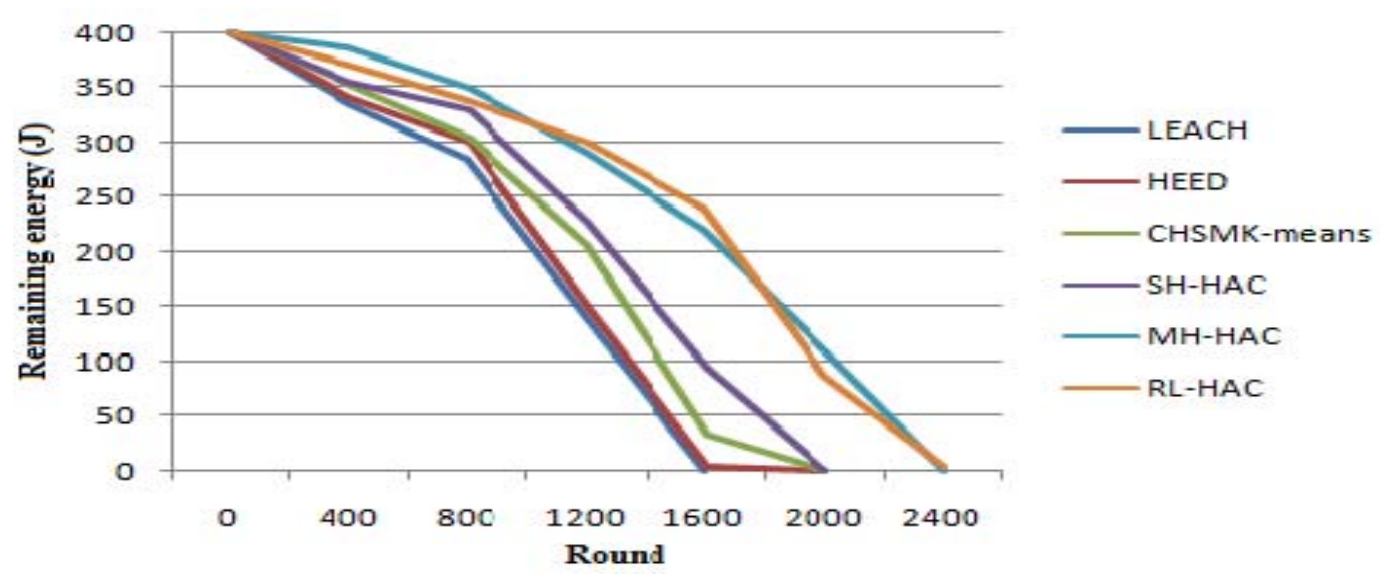

Figure 7. Residual energy in the network

Figure 7 shows that the residual energy, which is the sum of residual energy over all the sensors, is significantly higher with MH-HAC and RL-HAC than other clustering schemes. These two schemes achieve a gain in network lifetime of 33\% than LEACH and HEED and 16\% CHSMK-means. Moreover, at the same round where all the sensors deplete their energy with LEACH and HEED, about $62 \%$ of energy still available with MH-HAC and RL-HAC. Hence, the double effect of these two schemes, through efficient cluster formation and multi-hop communication to the BS, is quite noticeable. Even, SH-HAC is shown to perform slightly better than other single-hop schemes. The same trends can be found in Figure 8, where we depict the number of alive nodes in the network.

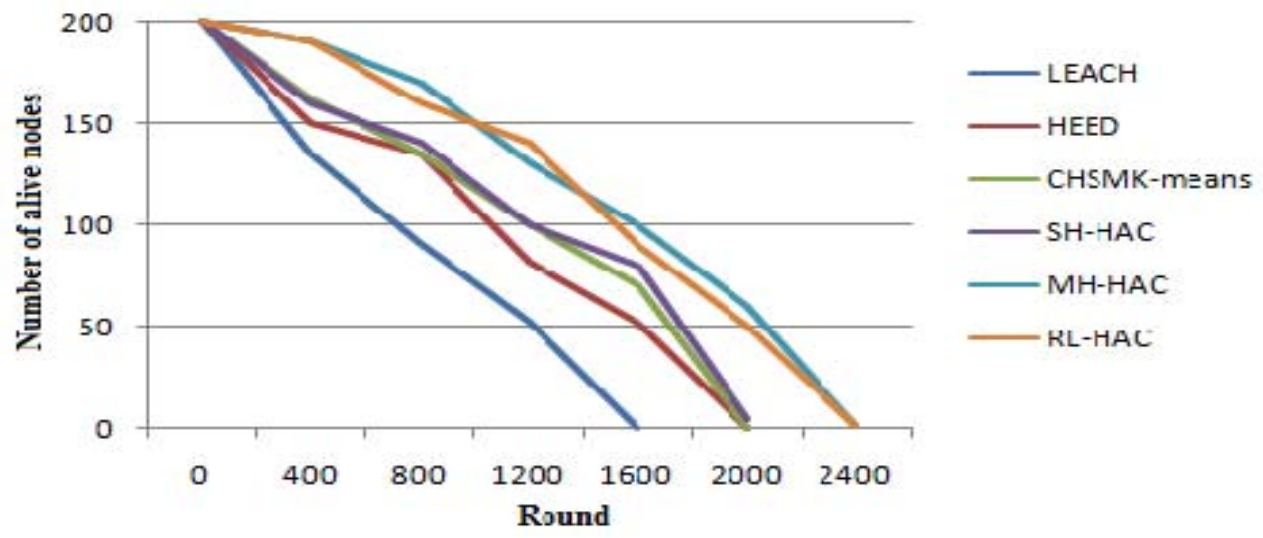

Figure 8. Number of alive nodes in the network

The total number of transmitted packets with MH-HAC and RL-HAC by varying the network area and the number of sensors are presented in Table 3. We can see that the results are quite close, but RLHAC performs slightly better than $\mathrm{MH}-\mathrm{HAC}$ for large number of sensors. This is a not trivial result as the Steiner tree built with Algorithm 3 is not optimal.

One reason is that RL-HAC can ask other nodes than the $\mathrm{CHs}$ to forward data traffic to the BS. The other reason is that the energy consumption exhibit a quartic growth when the transmission distance 
exceeds $d_{0}$, as stated in (4), whereas the edge weights provided to the minimum spanning tree increase only linearly with the distance. Hence, there is a beneficial side-effect on the energy consumption in restricting the transmission range.

Table 3. Comparisons between our clustering schemes

\begin{tabular}{|c|c|c|c|}
\hline Network area & Number of sensors & MH-HAC & RL-HAC \\
\hline \multirow{3}{*}{$100 \mathrm{~m} \times 100 \mathrm{~m}$} & 100 & 371558 & 375584 \\
\hline & 500 & 9864306 & 9838465 \\
\hline & 1000 & 39529227 & 39694730 \\
\hline \multirow{3}{*}{$500 m \times 500 m$} & 100 & 45306 & 49649 \\
\hline & 500 & 1901557 & 1875157 \\
\hline & 1000 & 7560203 & 7854365 \\
\hline \multirow{3}{*}{$1000 \mathrm{~m} \times 1000 \mathrm{~m}$} & 100 & 27154 & 23733 \\
\hline & 500 & 1149328 & 1124413 \\
\hline & 1000 & 5144873 & 5265602 \\
\hline
\end{tabular}

\section{Conclusion}

In this paper, the several clustering and communication schemes to improve energy-efficiency of WSNs and hence increase their lifetime were proposed. In contrast to some exiting schemes, our clustering approach based on HAC determines the most suitable number of clusters with regard to energy consumption. Furthermore, we designed a novel cluster head selection mechanism based on the proximity from a virtual energy-aware centroid. As this virtual point can move during the operation of the network, the $\mathrm{CH}$ role can be handed over to other nodes without shifting the cluster membership. Single-hop and multi-hop communication between the $\mathrm{CHs}$ and the base station have been also studied in this paper.

Numerical experiments have shown that multi-hop routing protocols on the overlay nodes lead to substantial lifetime gains compared to LEACH, HEED and K-means. But even our single-hop has exhibited better performance than these protocols due to the clustering scheme and the $\mathrm{CH}$ selection and re-selection mechanisms. As future work we plan to design a fully distributed version of our clustering and communication schemes in order to improve the scalability and the dependability of our approach.

\section{References}

1. 10 Emerging Technologies That Will Change. The World. Technology review February 2003.

2. Chen, H., Wu, C. S., Chu, Y. S., Cheng, C. C. and Tsai, L. K. (2007) Energy Residue Aware (ERA) Clustering Algorithm for Leach-Based Wireless Sensor Networks. In: Proceedings of the Second International Conference on Systems and Networks Communications (ICSNC 2007), August 25-31, 2007, Cap Esterel, French Riviera, France, page 40, 2007.

3. Dong, Z. and Chun, C. (2012) Hybrid Communication Method for Data Gathering in Wireless Sensor Networks. In: Proceedings of Springer International Conference on Information Technology and Management Science (ICITMS 2012).

4. Du, T., Qu, S., Liu, F. and Wang, Q. (2015) An Energy Efficiency Semi-Static Routing Algorithm for $\{$ WSNs $\}$ Based on $\{$ HAC $\}$ Clustering Method. Information Fusion, 21:18 - 29, 2015.

5. Heinzelman, W. B., Chandrakasan, A. P., and Balakrishnan, H. (2002) An Application-Specific Protocol Architecture for Wireless Microsensor Networks. Trans. Wireless. Comm., 1(4):660-670, October 2002.

6. Kou, L., Markowsky, G. and Berman, L. (1981) A Fast Algorithm for Steiner Trees. Acta Informatica, 15(2):141-145, 1981.

7. Lukasová, A. (1979) Hierarchical Agglomerative Clustering Procedure. Pattern Recognition, 11(56):365-381, 1979.

8. Lung, C. H and Zhou, C. (2010). Using Hierarchical Agglomerative Clustering in Wireless Sensor Networks: An Energy-Efficient and Flexible Approach. Ad Hoc Networks, 8(3):328-344, 2010.

9. Manjeshwar, A. and Agrawal, D. P. (2001) TEEN: Arouting Protocol for Enhanced Efficiency in Wireless Sensor Networks. In: Proceedings of the 15th International Parallel \& Distributed Processing Symposium (IPDPS-01), San Francisco, CA, April 23-27, 2001, page 189, 2001.

10. Park, G.Y., Kim, H., Jeong, H. W., and Youn, H. Y. (2013) A Novel Cluster Head Selection Method Based on K-Means Algorithm for Energy Efficient Wireless Sensor Network. In: Proceedings of the 2013 27th International Conference on Advanced Information Networking and Applications Workshops, (WAINA '13), pages 910-915, Washington, DC, USA, 2013. IEEE Computer Society. 
11. Robins, G. and Zelikovsky, A. (2005) Tighter Bounds for Graph Steiner Tree Approximation. SIAM J. Discrete Math., 19(1):122-134, 2005.

12. WeiWang, G., Zhang, C. X. and Zhuang, J. (2014) Clustering with Prim's Sequential Representation of Minimum Spanning Tree. Applied Mathematics and Computation, 247:521-534, 2014.

13. Youn H. Y. and Kim, K. T. (2005) PEACH: Proxy-Enable Adaptive Clustering Hierarchy for Wireless Sensor Networks. In: Proceedings of the 2005 International Conference on Wireless Networks, (ICWN 2005), Las Vegas, Nevada, USA, June 27-30, 2005, pages 52-56, 2005.

14. Younis, O. and Fahmy, S. (2004) HEED: A Hybrid, Energy-Efficient, Distributed Clustering Approach for Ad Hoc Sensor Networks. IEEE Trans. Mob. Comput., 3(4):366- 379, 2004. 